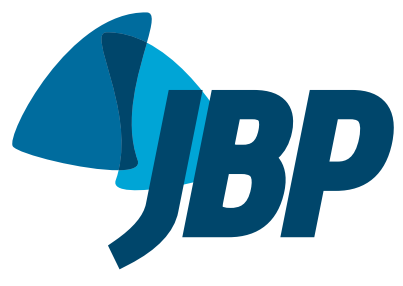

\title{
Evaluation of the gauge of needles used in the collection of specimens during endobronchial ultrasound-guided transbronchial needle aspiration
}

\author{
Goohyeon Hong',a, Ji Hae Koo ${ }^{2, b}$
}

\section{TO THE EDITOR:}

Endobronchial ultrasound-guided transbronchial needle aspiration (EBUS-TBNA) is a minimally invasive procedure that is widely used to sample mediastinal lesions, hilar lesions, and lesions adjacent to the central airway. ${ }^{(1,2)}$ EBUS-TBNA was originally performed using dedicated 22-gauge needles. Recently, 21-gauge needles have been employed to improve the quality of biopsy specimens. The relative sampling utility of 21-gauge needles, in comparison with that of 22-gauge needles, remains controversial.

In the present study, we assessed the adequacy of histological specimens and the cellularity of cytological specimens obtained with 21- and 22-gauge needles. We analyzed data related to patients referred to a university hospital for EBUS-TBNA between 2014 and 2016. We included consecutive patients with hilar/ mediastinal lymphadenopathy or tumors adjacent to the central airway. EBUS-TBNA was performed under local anesthesia with light conscious sedation with a convexprobe ultrasound bronchoscope (BF-UC260FW; Olympus, Tokyo, Japan) and a dedicated ultrasound scanner (EU-ME1; Olympus). All procedures were performed by the same pulmonologist. The needle gauge used was at the discretion of the operator, who employed either 21-gauge or 22-gauge dedicated needles (ViziShot, NA-201SX-4021 or NA-201SX-4022; Olympus). Only one sampling needle was used for each patient. Rapid on-site cytology evaluation was not performed.

The cytological and histological quality of needle aspirates was assessed by an experienced pathologist blinded to the needle gauge used. Each histological specimen was evaluated separately and categorized as follows: class I, diagnostic; class II, non-diagnostic but adequate; or class III, non-diagnostic and inadequate. In brief, specimens of classes I and II were considered adequate, either allowing a specific diagnosis to be made or at least containing elements indicating that the target lesion had been sampled. Cellularity was graded as follows: $A$, high $(60-100 \%) ; B$, moderate $(30-59 \%) ; C$, low $(5-30 \%) ; D$, scant $(<5 \%)$; or $\mathrm{E}_{1}$ none (no lymphoid cells). Specimens of grades A, B, and $\mathrm{C}$ were considered adequate.

We evaluated 115 lesions from 68 cases (59 male patients and 9 female patients). The mean age of the patients was 63.5 years (range, 27-84 years). Based on the histological analysis, 57 patients had malignancies and 11 had benign lesions. A total of 57 lesions (in 36 patients) were punctured by 21-gauge needles, and 58 lesions (in 32 patients) were punctured by 22-gauge needles. The mean number of lesions punctured per patient was 1.69 (range, 1-3) and the mean total passes per patient was 4.20 (range, 2-9). The mean short axis diameter of the targeted lesions was $13.5 \mathrm{~mm}$ (range, 5-53). We found no significant between-group differences in terms of gender, age, prevalence of primary malignancy, lesion size, location, or number of needle passes.

Of the 57 lesions punctured by 21-gauge needles, $50.9 \%$ yielded class I specimens, $31.6 \%$ yielded class II specimens, and $17.5 \%$ yielded class III specimens. Of 58 lesions punctured by 22 -gauge needles, $46.6 \%$ yielded class I specimens, $32.8 \%$ yielded class II specimens, and $20.6 \%$ yielded class III specimens. In the $21-$ and 22-gauge groups, adequate histological specimens were obtained in $82.5 \%$ and $79.4 \%$ of the procedures, respectively, a difference that was not significant ( $p=$ 0.81 ). Of the 57 specimens obtained with 21 -gauge needles, $77.1 \%$ showed adequate cellularity and $22.9 \%$ did not, compared with $55.2 \%$ and $44.8 \%$, respectively, of the 58 specimens obtained with 22-gauge needles. The cytological adequacy of the specimens obtained with 21-gauge needles was significantly higher than was that of those obtained with 22-gauge needles ( $p$ $=0.018$ ). The histological classes, cytological grades, and qualities of the specimens are summarized in Table 1. We observed no major complications in either group.

In our study, 21-gauge needles were found to be superior to 22-gauge needles in terms of providing high-quality cytological specimens, although the adequacy of the histological specimens did not differ between the two groups. Six studies have assessed the effects of needle gauge in EBUS-TBNA, ${ }^{(3-8)}$ although the results remain controversial. Saji et al. ${ }^{(3)}$ reported that diagnostic accuracy was significantly higher when a 21-gauge needle was used than when a 22-gauge needle was used, and use of the former greatly improved the diagnosis of malignancy. Jeyabalan et al. (7) found that 21-gauge needles were superior to 22-gauge needles in terms of histopathological assessment of benign lesions (especially sarcoidosis) and malignant mediastinal lymphadenopathy. Nakajima et al. ${ }^{(5)}$ reported that the number of cells in cytological specimens was significantly greater when 21-gauge needles were used than when

1. Division of Pulmonary and Critical Care Medicine, Department of Internal Medicine, Dankook University Hospital, Dankook University College of Medicine, Cheonan, South Korea. 
Table 1. Classification of the specimens obtained by endobronchial ultrasound-guided transbronchial needle aspiration. ${ }^{a}$

\begin{tabular}{|c|c|c|c|}
\hline \multirow[t]{2}{*}{ Variable } & \multicolumn{2}{|c|}{$\begin{array}{c}\text { EBUS-TBNA } \\
\text { Needle size }\end{array}$} & \multirow[t]{2}{*}{$\mathbf{p}^{*}$} \\
\hline & $\begin{array}{l}\text { 21-gauge } \\
(N=57)\end{array}$ & $\begin{array}{l}\text { 22-gauge } \\
(N=58)\end{array}$ & \\
\hline \multicolumn{4}{|l|}{ Histological specimen class } \\
\hline I. Diagnostic & $29(50.9)$ & $27(46.6)$ & $0.81^{\dagger}$ \\
\hline II. Non-diagnostic but adequate & $18(31.6)$ & $19(32.8)$ & \\
\hline III. Non-diagnostic and inadequate & $10(17.5)$ & $12(20.6)$ & \\
\hline \multicolumn{4}{|l|}{ Cytological specimen grade $^{b}$} \\
\hline A. High & $10(17.5)$ & $8(13.8)$ & $0.018^{\ddagger}$ \\
\hline B. Moderate & $24(42.1)$ & $13(22.4)$ & \\
\hline C. Low & $10(17.5)$ & $11(19.0)$ & \\
\hline D. Scant & $3(5.4)$ & $14(24.1)$ & \\
\hline E. None & $10(17.5)$ & $12(20.7)$ & \\
\hline
\end{tabular}

EBUS-TBNA: endobronchial ultrasound-guided transbronchial needle aspiration. ${ }^{\text {DData }}$ are presented as $\mathrm{n}(\%)$. ${ }^{\mathrm{b}} \mathrm{A}$, high $(60-100 \%) ; B$, moderate $(30-59 \%) ; C$, low $(5-30 \%) ; \mathrm{D}$, scant $(<5 \%)$; or $\mathrm{E}$, none (no lymphoid cells). * Fisher's exact test. ${ }^{+}$Classes I and II vs. class III. ${ }^{\ddagger}$ Grades $A, B$, and $C$ vs. grades D and $\mathrm{E}$.

22-gauge needles were used, and the extent of histological preservation was significantly greater when the former were used in order to sample malignant lesions. Oki et al. ${ }^{(4)}$ reported that no difference in sampling yield between 21- and 22-gauge needles, both of which afforded good yields. Yarmus et al. ${ }^{(6)}$ performed a retrospective multicenter (six-center) study and found that neither the diagnostic yield nor sample adequacy differed according to the gauge of needle used, although significantly fewer passes were required when 21 -gauge needles were used than when 22-gauge needles were used, supporting the idea that the use of the former can increase the quantity of core tissue obtained and the extent of histological preservation. Most recently, Muthu et al. ${ }^{(8)}$ assessed the diagnostic yield and adequacy (granuloma density) of histological specimens obtained with 21- and 22-gauge needles from patients with sarcoidosis. The authors found no difference between the two groups in terms of diagnostic yield or adequacy of the aspirate.

Theoretically, a larger-diameter (21-gauge) needle provides samples of higher volume. ${ }^{(9)}$ However, we found no significant differences between 21- and 22-gauge EBUS-TBNA needles in terms of the diagnostic yield or adequacy of histological specimens, which is in keeping with the findings of previous studies. ${ }^{(4-6,8)}$ That might be attributable to the inner diameters of both needles being sufficiently large to allow adequate histological sampling of core tissue. ${ }^{(10)}$ In addition, because multiple lymph nodes are generally sampled in multiple passes, the amount of histological material obtained is usually diagnostically adequate. Although the adequacy of histological specimens obtained using either needle gauge has been debated, a trend toward improved sample adequacy when 21-gauge needles are used was apparent in our study. In particular, the quality of cytological specimens obtained with 21-gauge needles was significantly superior to that of those obtained with 22-gauge needles. Although gross and macroscopic specimen collection was easier when 21-gauge needles were employed, 22-gauge needles are especially suitable for EBUS-TBNA. Such needles have soft sheaths, which improves convex probe EBUS flexion even when the needle is inside the working channel. That facilitates EBUS-TBNA and allows acquisition of samples from nodes in the more distal parts of the airways.

In summary, we found no significant difference between 21- and 22-gauge EBUS-TBNA needles in terms of the adequacy of the histological specimens obtained. However, 21-gauge needles were superior in terms of providing adequate cytological specimens.

\section{ACKNOWLEDGMENTS}

The present study was supported by the research fund of Dankook University in 2018.

\section{REFERENCES}

1. Adams K, Shah PL, Edmonds L. Lim E. Test performance of Adams K, Shah PL, Edmonds L. Lim E. Test performance of endobronchial ultrasound and transbronchial needle aspiration biopsy for mediastinal staging in patients with lung cancer: systematic review and metaanalysis. Thorax. 2009;64(9):757-62. https://doi.org/10.1136/ thx.2008.109868

2. Varela-Lema L, Fernández-Villar A, Ruano-Ravina A. Effectiveness and safety of endobronchial ultrasound-transbronchial needle aspiration: a systematic review. Eur Respir J. 2009:33(5):1156-64. https://doi.org/10.1183/09031936.00097908

3. Saji J, Kurimoto N, Morita K, Nakamura M, Inoue T, Nakamura H, et

al. Comparison of 21-gauge and 22-gauge Needles for Endobronchia Ultrasound-Guided Transbronchial Needle Aspiration of Mediastinal and Hilar Lymph Nodes. J Bronchology Interv Pulmonol. 2011;18(3):239-46. https://doi.org/10.1097/LBR.0b013e3182273b41

4. Oki M, Saka H, Kitagawa C, Kogure Y, Murata N, Ichihara S, et al Randomized Study of 21-gauge Versus 22-gauge Endobronchia Ultrasound-guided Transbronchial Needle Aspiration Needles for Sampling Histology Specimens. J Broncholology Interv Pulmonol. 2011;18(4):306-10. https://doi.org/10.1097/LBR.0b013e318233016c

5. Nakajima T, Yasufuku K, Takahashi R, Shingyoji M, Hirata T, Itami M et al. Comparison of 21-gauge and 22-gauge aspiration needle during 
endobronchial ultrasound-guided transbronchial needle aspiration Respirology. 2011;16(1):90-4. https://doi.org/10.1111//.14401843.2010.01871.x

6. Yarmus LB, Akulian J, Lechtzin N Yasin F, Kamdar B, Ernst A, et al. Comparison of 21-gauge and 22-gauge aspiration needle in endobronchial ultrasound-guided transbronchial needle aspiration: results of the American College of Chest Physicians Quality Improvement Registry, Education, and Evaluation Registry. Chest. 2013;143(4):1036-1043. https://doi.org/ 10.1378/chest.12-1205

7. Jeyabalan A, Shelley-Fraser G, Medford AR. Impact of needle gauge on characterization of endobronchial ultrasound-guided transbronchial needle aspiration (EBUS-TBNA) histology samples. Respirology. 2014;19(5):735-9. https://doi.org/10.1111/resp.12293

8. Muthu V, Gupta N, Dhooria S, Sehgal IS, Bal A, Aggarwal AN, et al. A Prospective, Randomized, Double-Blind Trial Comparing the Diagnostic Yield of 21- and 22-Gauge Aspiration Needles for Performing Endobronchial Ultrasound-Guided Transbronchial Needle Aspiration in Sarcoidosis. Chest. 2016;149(4):1111-3. https://doi. org/10.1016/j.chest.2016.01.014

9. Song TJ, Kim JH, Lee SS, Eum JB, Moon SH, Park DY, et al. The prospective randomized, controlled trial of endoscopic ultrasoundguided fine-needle aspiration using $22 \mathrm{G}$ and $19 \mathrm{G}$ aspiration needles for solid pancreatic or peripancreatic masses. Am J Gastroenterol. 2010;105(8):1739-45. https://doi.org/10.1038/ajg.2010.108

10. Yasufuku K, Chiyo M, Sekine $Y$, Chhajed PN, Shibuya K, lizasa $T$, et al. Real-time endobronchial ultrasound-guided transbronchial needle aspiration of mediastinal and hilar lymph nodes. Chest. 2004;126(1):122-8. https://doi.org/10.1378/chest.126.1.122 